\title{
Metabolic activity phenotyping of single cells with multiplexed vibrational probes
}

\author{
Zhilun Zhao, Chen Chen, Hanqing Xiong, Jingwei Ji, and Wei Min* \\ Department of Chemistry, Columbia University, New York, NY 10027 \\ Corresponding author: wm2256@columbia.edu
}




\section{Supplementary information}

\section{Batch to batch variation is the major source of variation for label-free Raman methods.}

Label-free methods such as Raman-based vibrational microscopy rely on physical and chemical properties to generate information about cell phenotypes ${ }^{1}$ and usually don't demand a priori knowledge of biomarkers. For this reason, label-free Raman spectroscopy has been extensively investigated and successfully demonstrated in many diagnostic applications ${ }^{2-4}$. In the past decade, great efforts have been put into improving the hardware in order to increase the signal to noise ratio ${ }^{5-7}$, and into developing sophisticated statistical tools such as machine learning algorithms to achieve better diagnostic results ${ }^{8}$. Yet challenges remain that hinder the prevalence of this powerful technique in broader applications. Among those challenges, to achieve a robust and reproducible detection and classification classifier model is the key.

Commonly the performance of the statistical model is validated by cross-validation. However, such an approach may suffer from model overfitting due to data leakage if batch to batch variation is the major source of variation, because the training and testing datasets are still from the same data pool even when they are derived from multiple batches ${ }^{9,10}$. To investigate this, we compared the model performance of three different data combination schemes (Fig. S6 $\mathrm{A}^{\sim} \mathrm{C}$ ) with label-free Raman spectroscopy. Three different cancer cell lines: A375, HeLa and MCF7 were used to train a model that is supposedly able to differentiate these three cell types. In this article, we refer a 'batch' of cells of a specific cell type to a dish of cells that are seeded on a particular day, and different 'batches' would mean that those cells were seeded on different days. Raman spectra of these batches of cells were put together to form the original data pool, from which an increasing number of samples were randomly withdrawn to construct the training set, and 100 samples that were not in the training set were randomly selected. Then a classifier model was trained with the training set and assessment was evaluated by using the trained classifier to predict the testing set (see material and methods for details). The process was repeated 100 times to ensure statistical significance.

To further validate the model, a random permutation test ${ }^{11}$ was carried out by firstly shuffling (permuting) the labels associated with each spectrum and then re-training the model using the shuffled dataset to make prediction in order to get the accuracy. Such a procedure was repeated 100,000 times to give the distribution of the accuracy under a null hypothesis. When the model from the real dataset is reasonable, the prediction accuracy from the 'false dataset' is expected to be low.

In scheme 1, only one batch of each cell type was used to form the original data pool, and the classifier was trained using randomly selected samples from the data pool (Fig. S6 A). The learning curve shows that as the training sample size increases the model prediction accuracy also increases until it reaches the plateau when the sample size is large enough ( 70 cells) (Fig. S6D, blue line). At this point, the trained model has a high accuracy predicting the testing data ( 98\%). The permutation test shows that if the labels of the samples are randomly permuted, the model would fail to predict, ruling out the possibility of false positive $^{11}$ (Fig. S6G). In scheme 2, at least 3 batches spanning months of each cell type were used to form the original data pool, from which the training and testing sets were randomly withdrawn (Fig. S6B, blue line). Similar to scheme 1, the model performance increases as the sample size grows. (Fig. S6E, blue line) However, the prediction accuracy reaches the plateau at $\sim 150$ cells, much later than it does in scheme1. 
Noticeable as well is that the best accuracy is still lower than that in scheme 1, hinting that more variation exists among batches. Likewise, the permutation test validates the model to rule out false positive (Fig. $\mathrm{S} 6 \mathrm{H}$ ). In scheme 3, the training set was randomly drawn from a data pool consisting of at least 3 batches of cells. The testing set, however, was selected randomly from a different data pool consisting of batches of cells that were not in the training set. This scheme mimicked a real-life application scenario where a classification model was first trained with existing data and then used to identify the unseen samples. The learning curve for this scheme shows, however, as the training size increases, the model does not improve, and never reaches a plateau of high accuracy even when the training set consists of thousands of samples (Fig. S6F, blue line). Cross-validation using the training set alone, on the other hand, shows a high model accuracy ( 90\%).

\section{Supplementary methods}

\section{Cell culture}

HeLa, MCF7, A375, MDA-MB-231 were cultured in Dulbecco's Modified Eagle's medium (DMEM: 4.5 g/L glucose; 11965, Gibco) supplemented with $10 \%$ Fetal bovine serum (FBS; 16000, Gibco) and 1\% antibiotics (Penicillin-Streptomycin-Glutamine; 10378016, Gibco). ZR-75-1 cells were cultured in RPMI 1640 medium (11875, Gibco) supplemented with 10\% Fetal bovine serum (FBS; 16000, Gibco) and 1\% antibiotics (Penicillin-Streptomycin-Glutamine; 10378016, Gibco). Cells were maintained in $5 \% \mathrm{CO}_{2}$ at $37^{\circ} \mathrm{C}$. All cells were passaged $2-3$ times a week and were never allowed to grow more than $90 \%$ confluency. Cells were tested mycoplasma negative.

HeLa cells were purchased from ATCC, MDA-MB-231 and A375 cells were kind gifts from Dr. Zeda Zhang at Memorial Sloan Kettering Cancer Center. MCF7 and ZR-75-1 cells were kind gifts from Dr. Sergey Pampou at Functional Genomics, High-Throughput Screening, Columbia Genome Center.

\section{Cell sample preparation}

We used freshly disassociated cells to fill the as much the illumination volume as possible in order to maximize Raman signal to noise ratio since disassociated cells were usually round and thicker.

For label-free samples, cells were first seeded in a 24 -well plate at $50 \%$ confluency with cell culture media. After overnight culture, cells were disassociated by TrypLE (12604, Gibco). Then cells were allowed to settle onto $18 \mathrm{~mm}$ round quartz coverslips (103302-258, Electron Microscopy Sciences) that were precoated with poly-lysine for $10 \mathrm{~min}$. Cells were subsequently washed twice with PBS (14040, Gibco) and fixed with 4\% freshly diluted paraformaldehyde (PFA; 15713S, Electron Microscopy Sciences), followed by 3 times wash with PBS. Double-sided adhesive imaging spacers (GBL654002, Sigma) were used to make 'sandwich' samples with glass slides.

For MAP samples, cells were first seeded in a 24-well plate at $50 \%$ confluency with cell culture media and allowed to adhere overnight. Then the culture media was replaced with MAP culture media (SI Table S1) and cells were further cultured in MAP media for 24 hours. For the drug tests, individual drugs (concentrations in SI Table S2) were added to the MAP media and cells were subject to the drug treatment for 24 hours. After the 24-hour culture, cell samples were prepared the same way as described above. 


\section{Automatic confocal micro-Raman Spectrometer}

The schematics of our home-built confocal Raman microscope is shown in Fig. 1A. We used a 532nm laser (Samba $532 \mathrm{~nm}, 400 \mathrm{~mW}$, Cobolt Inc.) as the light source. The laser beam was first collimated and expanded by the telescope lenses ( $L 1, L 2 / L 1^{\prime}, L_{2}$ ', Thorlabs). A half-wave plate (HWP, Thorlabs) and polarizing beamsplitters (PBS, Thorlabs) were used to switch between the two beam expansion ratios (L1, L2 or L1', L2') to achieve two different illumination spot sizes. For this article, the objective was underfilled and the illumination spot had a diameter of 8 um as the result. The expanded beam was then directed to the inverted microscope (IX71, Olympus) installed with a dichroic beamsplitter (LPD1, LPD02-532RU-25, Semrock). The emitted Raman signal first passed a pinhole (PH, 300um, Thorlabs) for background suppression and was relayed by two lenses (L3, L4, Thorlabs) before being projected to the spectrometer (Kymera 328i with 600 lines/mm grating blazed at 500 $\mathrm{nm}$, Andor). A long-pass filter (LP, LP03-532RU-25, Semrock) was installed between the relay lenses to block laser light from Rayleigh scattering. Raman signal was then collected by an EMCCD (Newton970, Andor). For brightfield imaging, a long-pass dichroic (LPD2, FF511-Di01, Semrock) was installed in front of the pinhole and a set of relay lenses ( $L 5, L 6)$ were used to project the brightfield images to the CMOS camera (DCC1645C, Thorlabs). A short-pass filter (SP) was installed to suppress ghost images.

In order to automatically acquire spectra from cells, a brightfield image was taken and the cells in the images were identified and their locations were subsequently calculated (Fig. S1 and S2). A motorized stage (HLD117, Prior) moved the sample slide so that the center of a cell aligned with the illumination spot, after which the spectrometer was triggered to acquire the spectrum. The procedure cycled until all cells within a field of view were taken. Background spectra were taken for the field of view for spectral background removal. The stage then moved to the next field of view and cycle through the procedures until the whole desired area was covered (Fig. S1 and S2). The process above was automated by a homebuilt software coded with Labview (National Instruments).

\section{Preprocessing}

We followed well established preprocessing protocols to preprocess the raw spectra ${ }^{12,13}$. Prior to the preprocessing procedures, the spectra were calibrated by 1:1 mixture of acetonitrile and toluene taken on the same day. After that, first, the quartz and water background were removed via an automatic algorithm ${ }^{14}$. Second, an iterative algorithm was used for baseline correction ${ }^{15}$. Third, the spectra were normalized to their amide peak. And lastly, the spectra were trimmed for subsequent analysis. The silent region $\left(2000-2300 \mathrm{~cm}^{-1}\right)$ was used for MAP analysis and fingerprint region $\left(600-1800 \mathrm{~cm}^{-1}\right)$ for labelfree analysis (Fig. S4).

\section{The supervised machine learning model}

In brief, once the training datasets were obtained, principal component analysis (PCA) was applied, followed by linear discrimination analysis (LDA). The k-nearest neighbor (k-NN) classifiers were then built on the dimension reduced data. To obtain the classifiers prediction accuracy, PCA and LDA were first applied to the testing dataset, then the trained classifiers were used to predict the labels of the test data, which were then compared to their known true labels to calculate the accuracy.

PCA has previously widely used to reduce noise and to improve statistical model robustness. By applying PCA to the training data, the training spectra were decomposed into multiple representations (scores and loading vectors) that capture as much of the information as possible. The number of components $\mathrm{n}$ was chosen in such a way that the accumulative variance explained was greater than $99 \%$. Then the top 
$\mathrm{n}$ PCA scores were used as the input of LDA, which is a supervised algorithm that maximizes the distance between group means while minimizing the variance within a group. LDA scores and projection vectors can be obtained by the algorithm, and LDA scores were further used for constructing k-NN clustering classifiers. To predict the test dataset, mean-centered spectra in the testing sets were first projected to PCA loading vectors obtained earlier in the training stage, and the PCA scores were further projected to training LDA projection vectors to obtain LDA scores for the testing sets. Then the dimension reduced data were input to the learned k-NN model to make the prediction. The model accuracies were then calculated by calculating the proportion of correctly predicted number of cells to the total number of cells. (Fig. S8)

\section{SI figures}

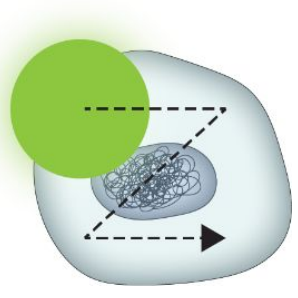

Figure S1 'Zig-zag' scanning mode to reduce variance for large cells. 
A
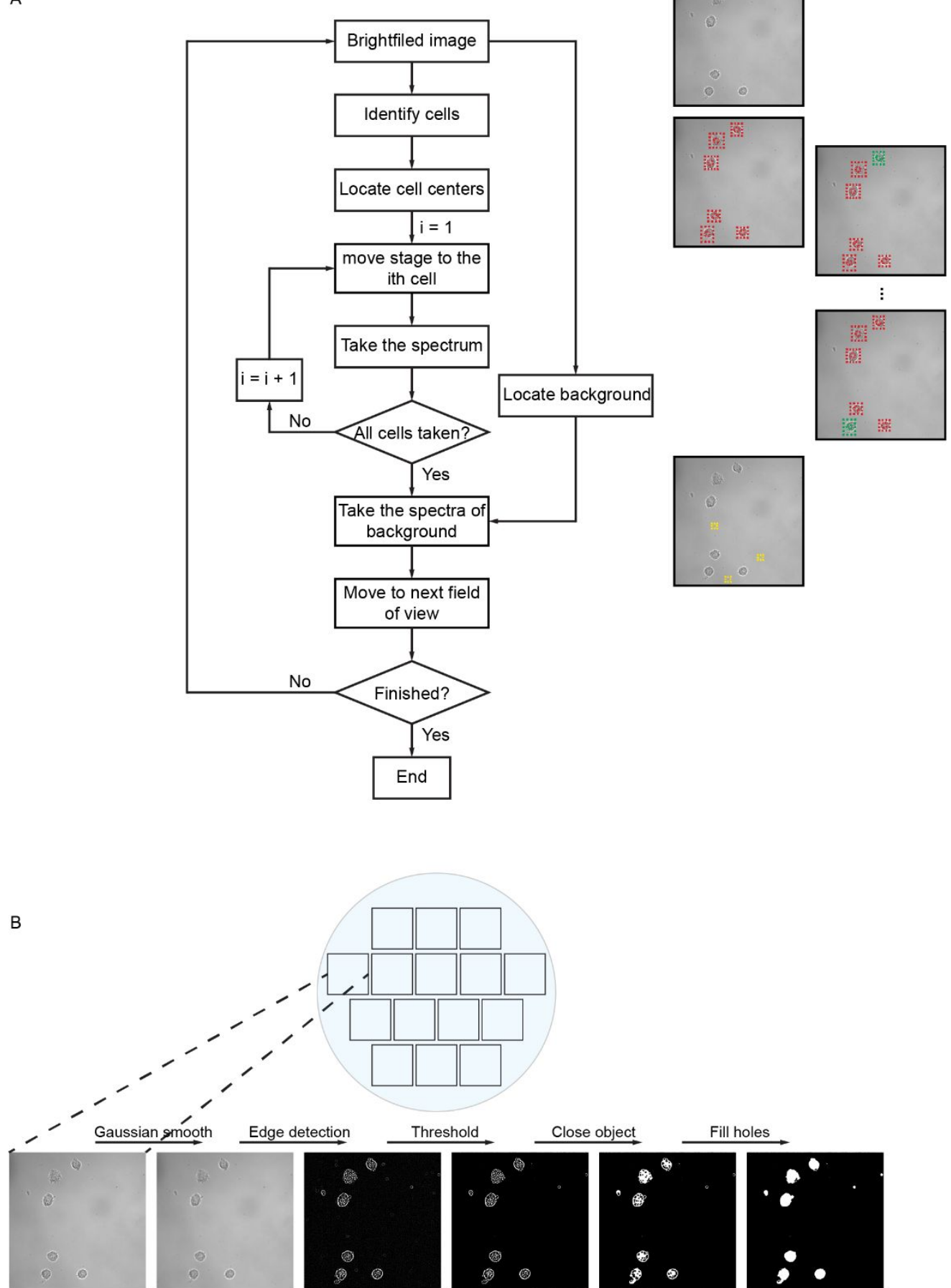

Convex hull Selection by size Remove objects on edges Find centers of mass
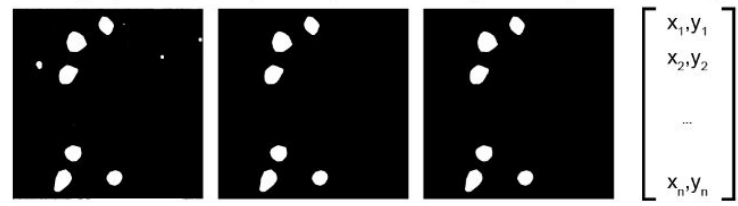

Figure S2 Software design principles of home-built Raman spectrometer. (A) Workflow of automatic Raman spectral acquisition for single cells. (B) Cell identification and localization algorithm. Machine vision to find the coordinates of the location of cell. 
A

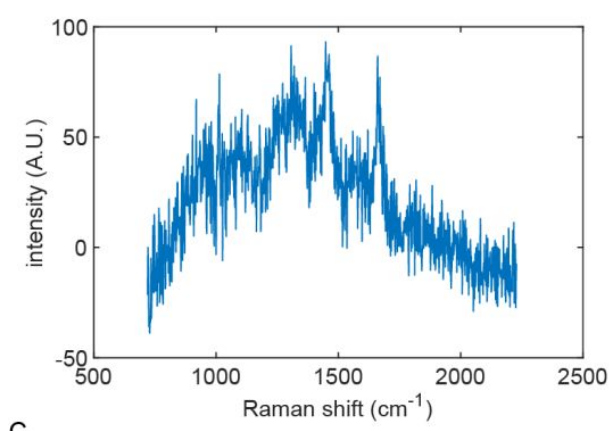

C

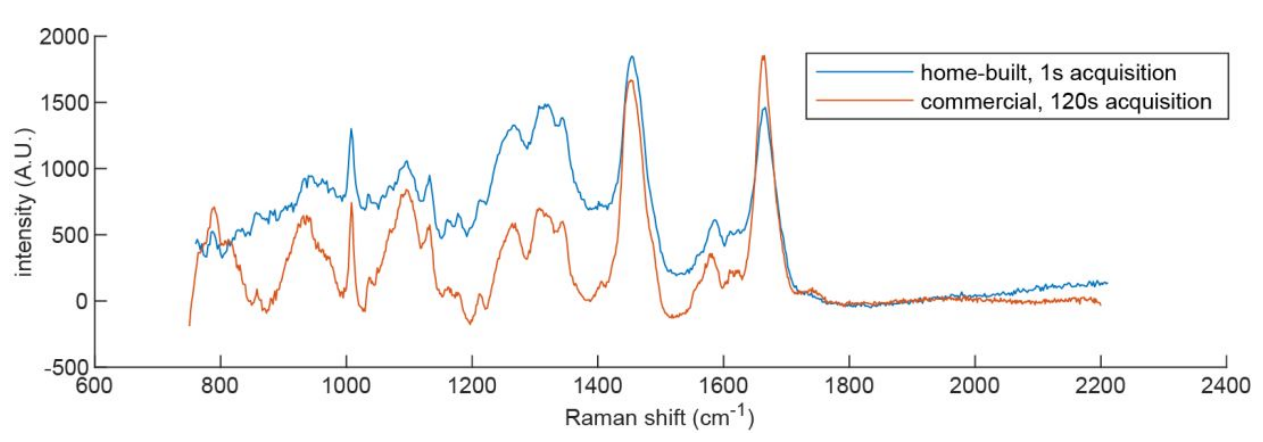

Figure S3 Signal comparison between commercially available micro-Raman spectrometer and homebuilt whole-cell confocal micro-Raman spectrometer. (A) A typical spectrum of a mammalian cell taken with Horiba XploRA PLUS micro-Raman spectrometer at 1s acquisition time. (B) A typical spectrum of a mammalian cell taken with home-built whole-cell confocal micro-Raman spectrometer at $1 \mathrm{~s}$ acquisition time. The signal to noise ratio (SNR) is at least 100 times greater than the spectrum taken with commercially available Raman spectrometer. (C) Normalized spectra taken with home-built Raman spectrometer and commercially available Raman spectrometer. To achieve same level of SNR, it usually takes 100 times longer exposure time with commercially available Raman spectrometer. Spectra with home-built Raman spectrometer were taken with 1s acquisition time; Spectra with commercial Raman spectrometer were taken with 120 s acquisition time. Spectra of 10 cells were averaged. 


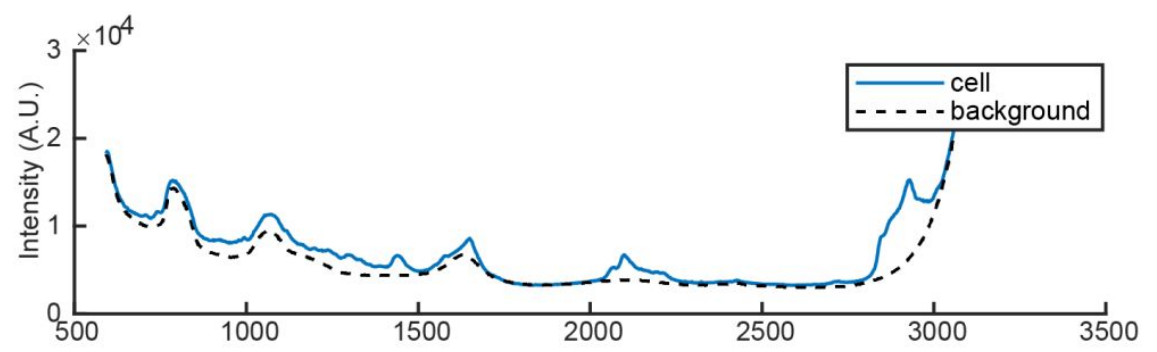

1. background removal

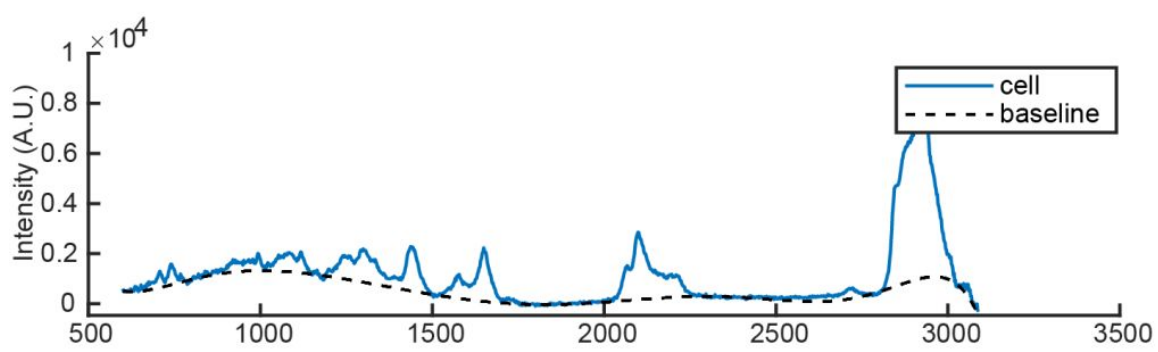

2. baseline correction

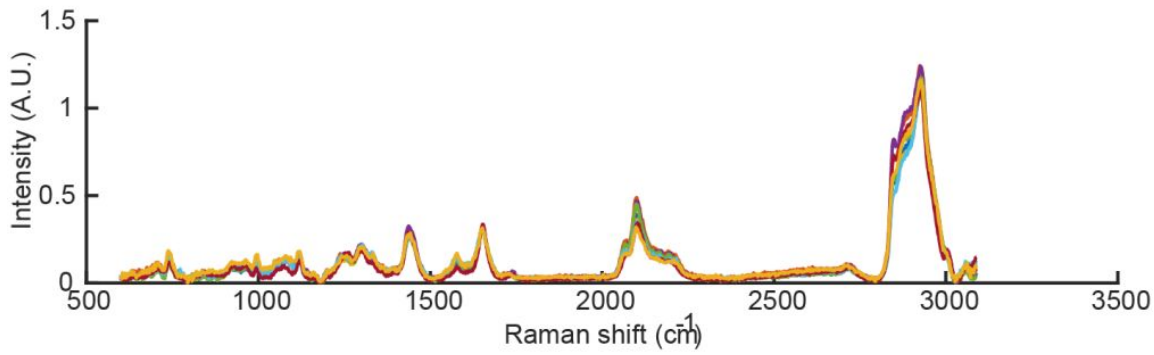

3. spectra normalization
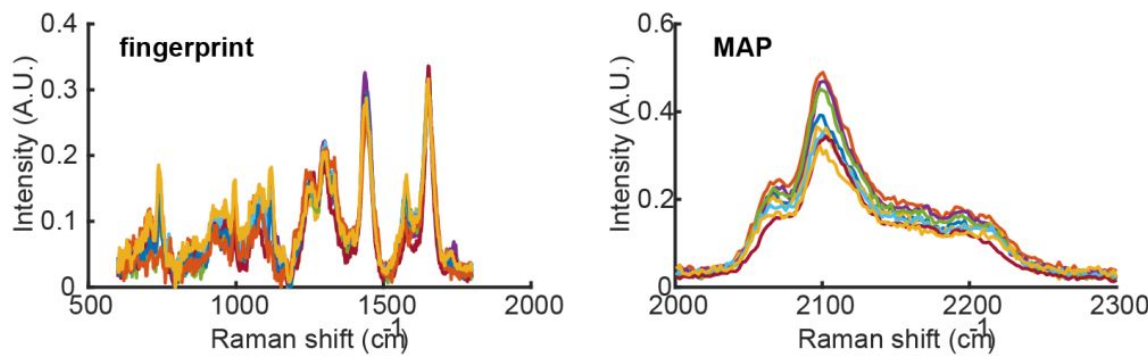

4. trim spectra

Figure S4 Spectral preprocessing procedures. 
A

B
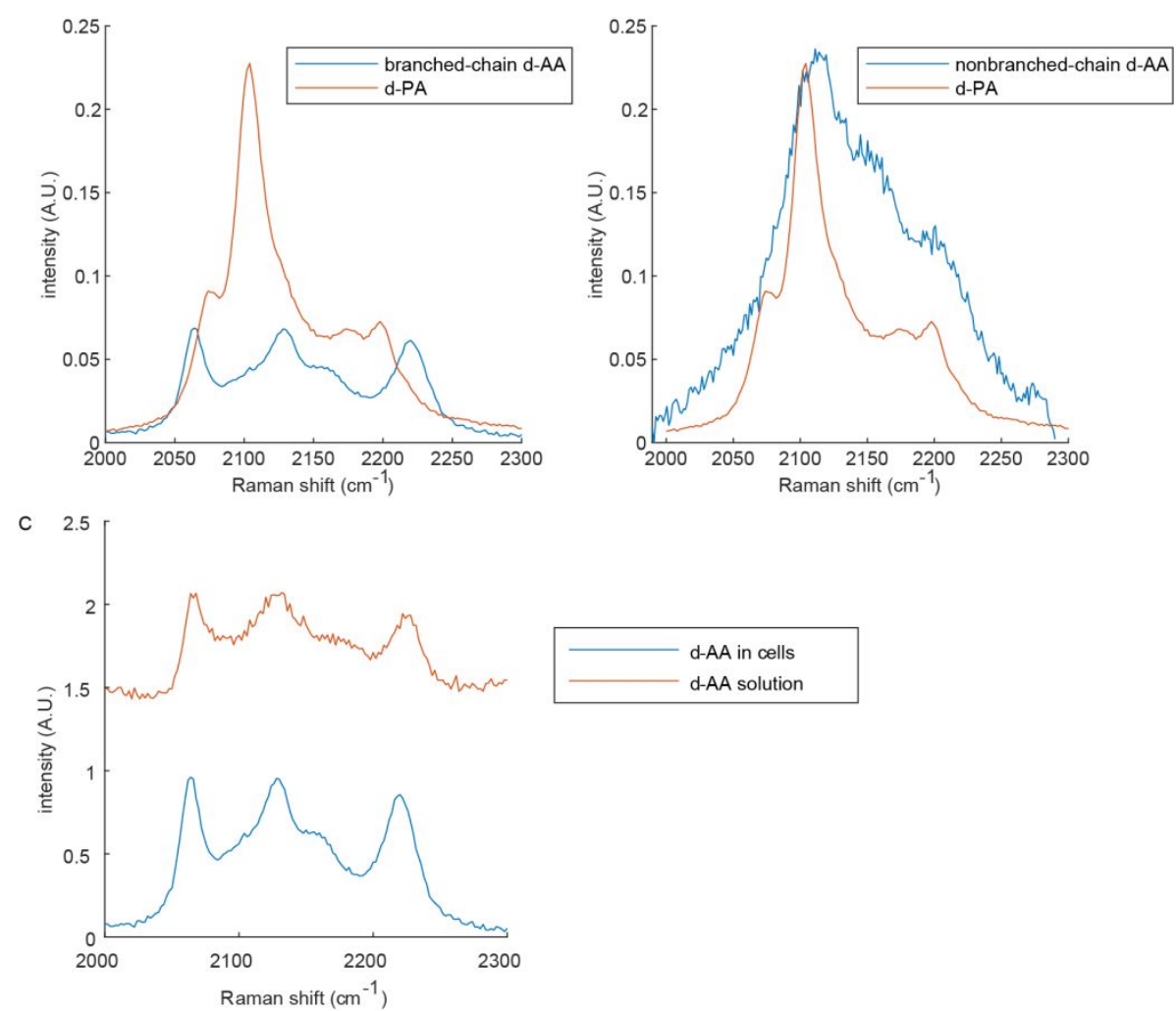

D

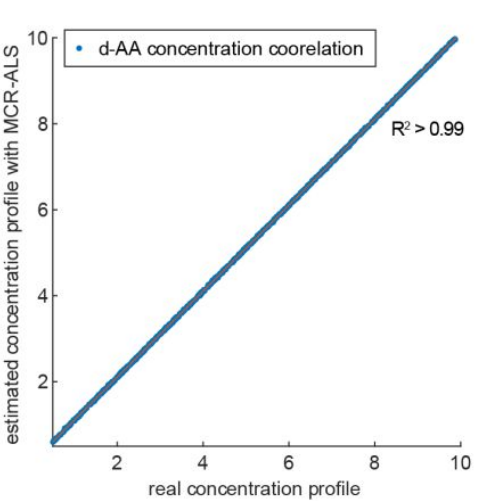

E

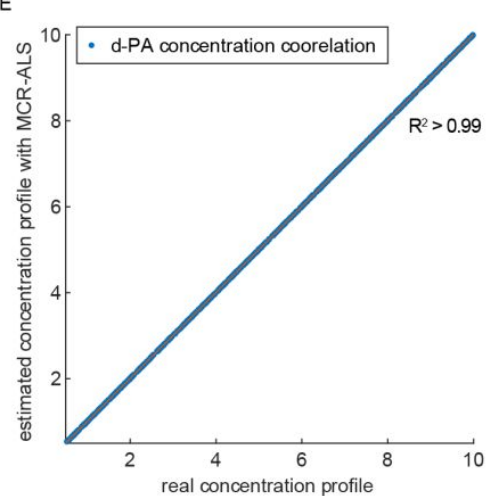

Figure S5 Spectra of deuterated branched-chain amino acids, deuterated nonbranched-chain amino acids and deuterated palmitic acid metabolites in cells. Deuterated branched-chain amino acids (A) are spectrally more resolvable to deuterated palmitic acid compared to deuterated nonbranched-chain amino acids (B). (B), Most of the spectra of deuterated nonbranched-chain amino acids overlap with that of deuterated palmitic acid, making it more difficult to spectral decompose the two. (C), Raman spectra of branched-chain d-AA solution and their metabolites in cells. The two spectra showed no significant difference, suggesting that d-AA were incorporated in cells mainly as building blocks in protein synthesis. (D) and (E), decomposition of d-AA and d-PA from simulated composite Raman spectra shows good accuracy ( $<2 \%$ overall error) with MCR-ACS. To construct composite Raman spectra, concentration profiles were randomly generated and then $6 \mathrm{~dB}$ noise was added ( $S N R=5)$. MCR-ACL was then used to decompose the simulated composite spectra and the resulting concentration profiles were compared with the real concentration. 
A

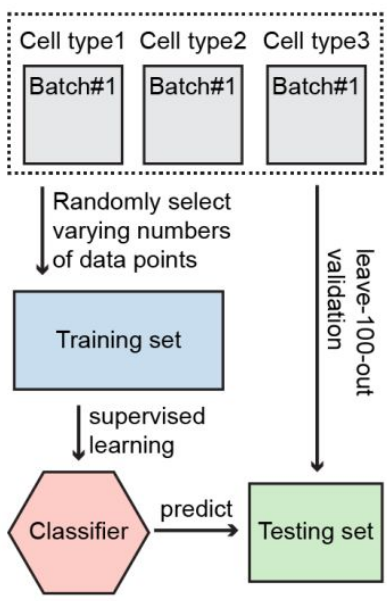

Scheme1
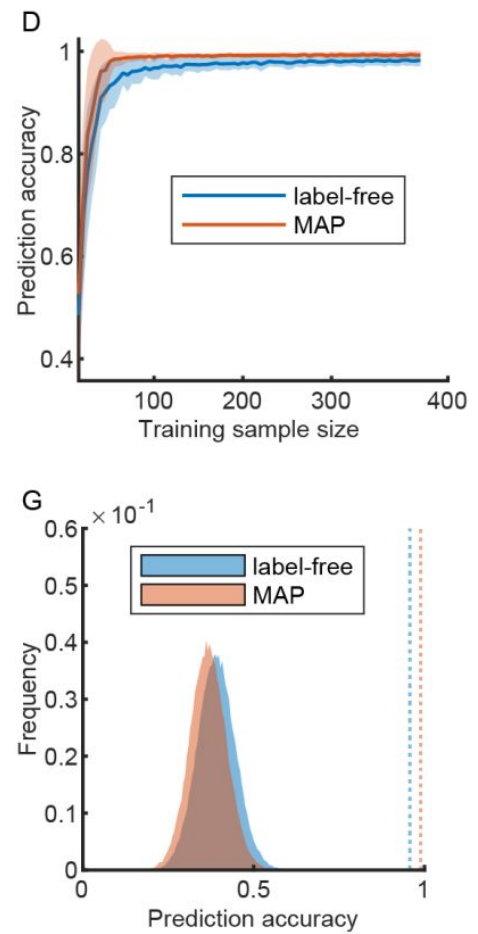

B

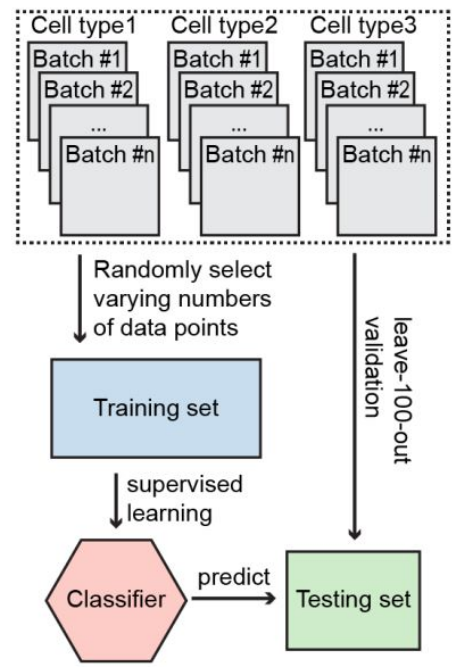

Scheme2

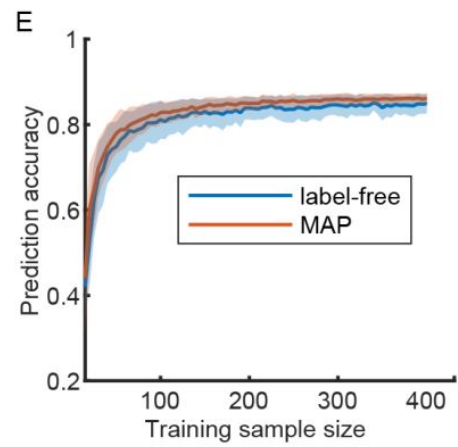

$\mathrm{H}$

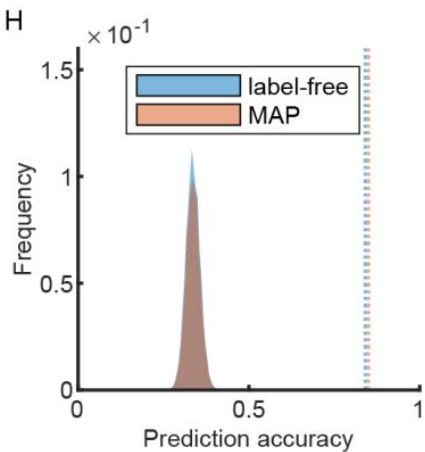

C

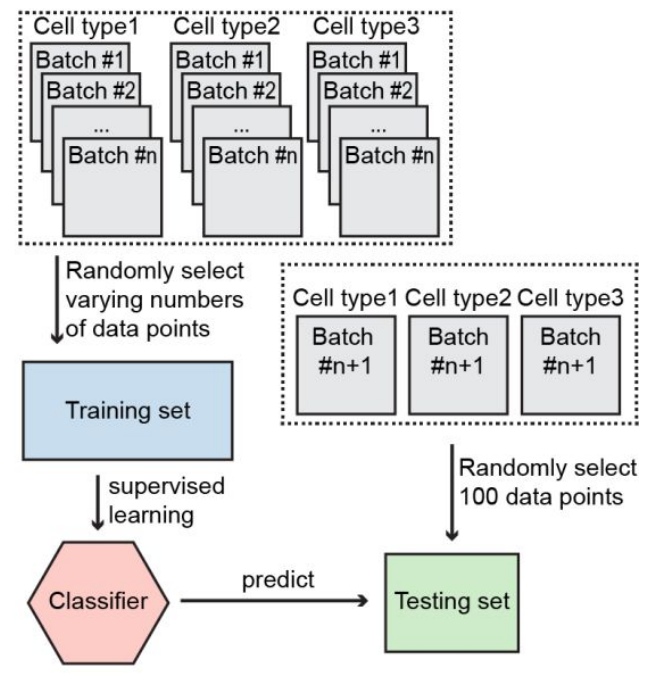

Scheme3

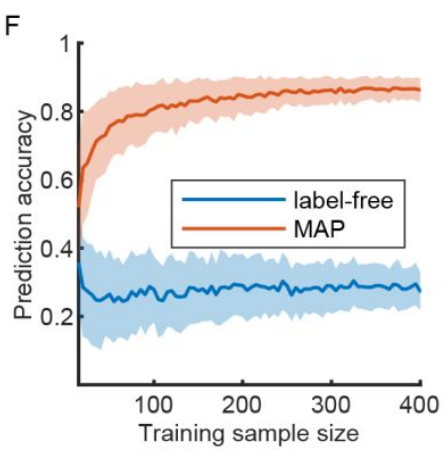

I

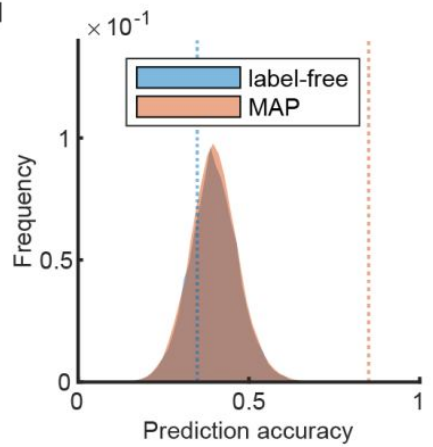

Figure S6 Classifier performance with three distinctive data combination schemes. $(A \sim C)$ Three approaches for constructing the datasets. $(D \sim F)$ Learning curves of the classifiers corresponding to scheme 1, 2, and 3 respectively. Blue lines, learning curves with label-free method; red lines, learning curves with MAP. Shaded areas represent standard deviation. (G I) Histograms of random permutation tests for scheme 1,2 and 3 respectively. The model accuracy of the permuted 'false dataset' is much lower than the true dataset (blue dash lines, label-free true accuracy; red dash lines, MAP true accuracy), rejecting the null hypothesis and confirming the high prediction accuracy from the true dataset is real. plane. 
A

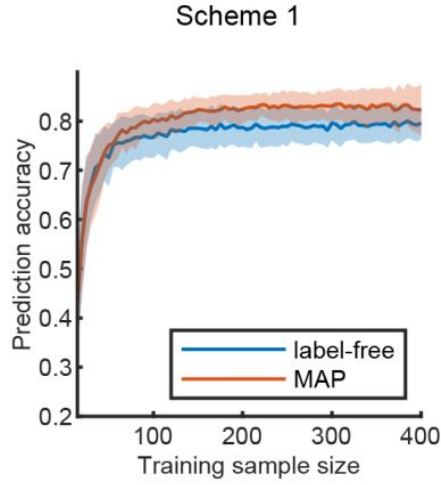

B

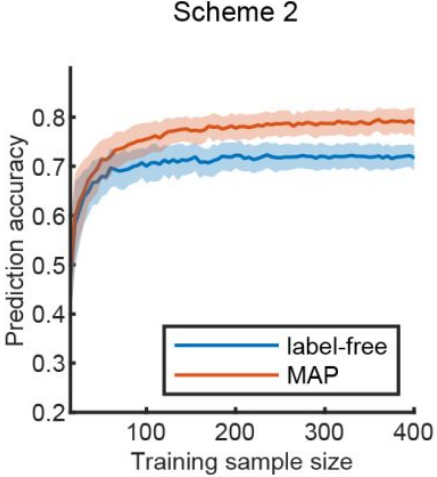

C

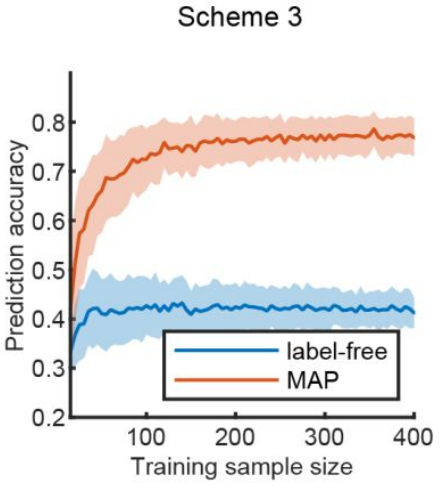

Figure S7 Model performance and discrimination of three human breast cancer cell lines. $(A \sim C)$, learning curves of classifiers corresponding to scheme 1,2 , and 3 respectively. Blue lines, learning curves with the label-free method; red lines, learning curves with MAP. Shaded areas indicate standard deviation.

scheme 1
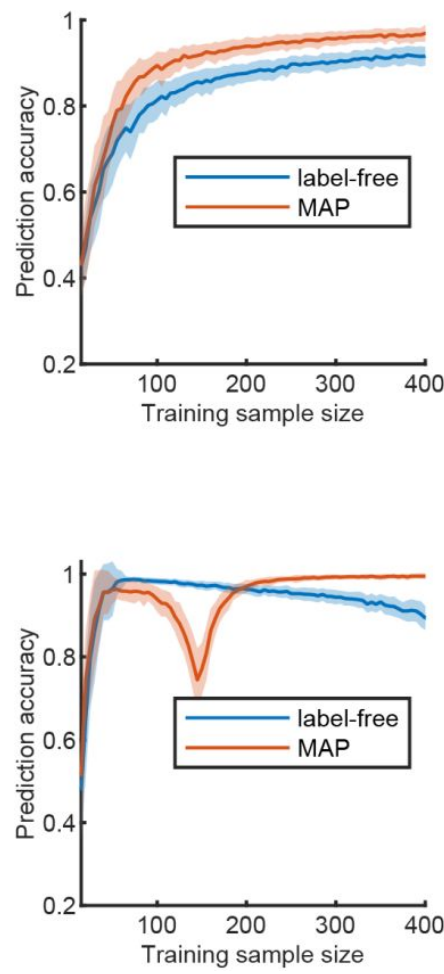

scheme 2
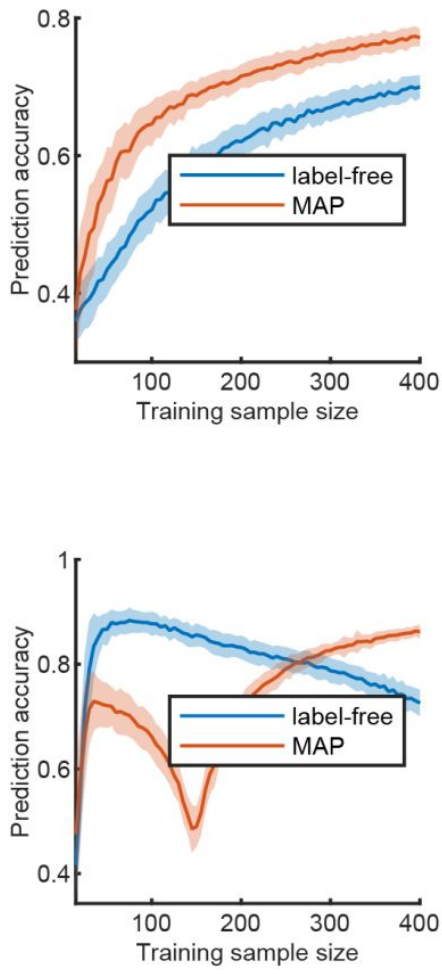

scheme 3
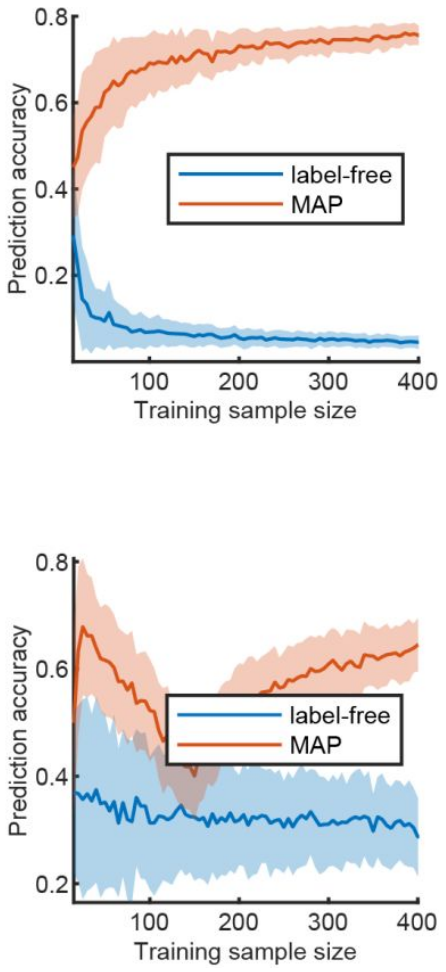

Figure S8 Learning curves of various statistical models. First row, classifiers were built with consecutive application of LDA and KNN without PCA. Second row, classifiers were built with consecutive application of PCA and KNN without LDA. 
Supplementary Table S1. MAP media formulation

\begin{tabular}{|c|c|}
\hline Components & Concentration (mg/L) \\
\hline \multicolumn{2}{|l|}{ Amino Acids } \\
\hline Glycine & 30 \\
\hline L-Arginine hydrochloride & 84 \\
\hline L-Cystine $2 \mathrm{HCl}$ & 63 \\
\hline L-Glutamine & 584 \\
\hline L-Histidine hydrochloride- $\mathrm{H} 2 \mathrm{O}$ & 42 \\
\hline L-Lysine hydrochloride & 146 \\
\hline L-Methionine & 30 \\
\hline L-Phenylalanine & 66 \\
\hline L-Serine & 42 \\
\hline L-Threonine & 95 \\
\hline L-Tryptophan & 16 \\
\hline L-Tyrosine disodium salt dihydrate & 104 \\
\hline \multicolumn{2}{|l|}{ Vitamins } \\
\hline Choline chloride & 4 \\
\hline D-Calcium pantothenate & 4 \\
\hline Folic Acid & 4 \\
\hline Niacinamide & 4 \\
\hline Pyridoxine hydrochloride & 4 \\
\hline Riboflavin & 0.4 \\
\hline Thiamine hydrochloride & 4 \\
\hline i-Inositol & 7.2 \\
\hline \multicolumn{2}{|l|}{ Inorganic Salts } \\
\hline Calcium Chloride (CaCl2) (anhyd.) & 200 \\
\hline Ferric Nitrate (Fe(NO3)3"9H2O) & 0.1 \\
\hline Magnesium Sulfate (MgSO4) (anhyd.) & 97.67 \\
\hline Potassium Chloride (KCl) & 400 \\
\hline Sodium Bicarbonate (NaHCO3) & 3700 \\
\hline Sodium Chloride ( $\mathrm{NaCl})$ & 6400 \\
\hline Sodium Phosphate monobasic (NaH2PO4-H2O & 125 \\
\hline \multicolumn{2}{|l|}{ Other Components } \\
\hline D-Glucose (Dextrose) & 4500 \\
\hline
\end{tabular}




\begin{tabular}{|l|l|}
\hline Fetal Bovine Serum, charcoal stripped & $10 \%$ \\
\hline Deuterated Vibrational probes & 105 \\
\hline L-Isoleucine-d10 & 105 \\
\hline L-Leucine-d10 & 94 \\
\hline L-Valine-d8 & $50 \mathrm{mM}$ \\
\hline palmitic acid-d31 &
\end{tabular}

Supplementary Table S2 Drug final concentrations used in this study

\begin{tabular}{|l|l|}
\hline cis-platin & $125 \mathrm{uM}$ \\
\hline blasticidin & $10 \mathrm{ug} / \mathrm{ml}$ \\
\hline cycloheximide & $100 \mathrm{uM}$ \\
\hline triacsin C & $5 \mathrm{uM}$ \\
\hline etoposide & $50 \mathrm{uM}$ \\
\hline
\end{tabular}

1. Joachim, W. E., Label-Free Monitoring of Cells in vitro. Springer: 2019.

2. Christoph, K.; Jürgen, P., The many facets of Raman spectroscopy for biomedical analysis. Anal. Bioanal. Chem. 2015, 407, 699-717.

3. Krafft, C.; Schmitt, M.; Schie, I. W.; Cialla-May, D.; Matthaus, C.; Bocklitz, T.; Popp, J., Label-Free Molecular Imaging of Biological Cells and Tissues by Linear and Nonlinear Raman Spectroscopic Approaches. Angew Chem. Int. Ed. Engl. 2017, 56 (16), 4392-4430.

4. Stöckel, S.; Kirchhoff, J.; Neugebauer, U.; Rösch, P.; Popp, J., The application of Raman spectroscopy for the detection and identification of microorganisms. J. Raman. Spectrosc. 2016, 47 (1), 89-109.

5. Kiselev, R.; Schie, I. W.; Askrabic, S.; Krafft, C.; Popp, J., Design and first applications of a flexible Raman micro-spectroscopic system for biological imaging. Biomed. Spectrosc. Ima. 2016, 5 (2), 115-127. 6. Yuta, S.; Koya, K.; Yoshifumi, W.; Dinghuan, D.; Shunji, T.; Chun-Jung, H.; Cheng, L.; Chia-Wei, S.; Hanqin, L.; Yasuhiro, F.; Sangwook, L.; Akihiro, I.; Yusuke, K.; Takeshi, H.; Shinya, S.; Fumihito, A.; Kenichi, K.; Hiroshi, T.; Mary, I.; Kei, H.; Takuro, I.; Misa, H.; Satoshi, M.; Kiyotaka, S.; Kanako, S.; Masako, N.; Masahiro, J.; Yutaka, Y.; Yaxiaer, Y.; Yo, T.; Takeaki, S.; Nao, N.; Keisuke, G.; Yasuyuki, O., Label-free chemical imaging flow cytometry by high-speed multicolor stimulated Raman scattering. Proc. Natl. Acad. Sci. U S A. 2019, 116, 15842-15848.

7. Yizhi, S.; Huabing, Y.; Wei, E. H., Raman activated cell sorting. Curr. Opin. Chem. Biol. 2016, 33, 1-8. 8. Jinchao, L.; Margarita, O.; Lorna, A.; Michael, F.; Christopher, J. S.; Stuart, J. G., Deep convolutional neural networks for Raman spectrum recognition: a unified solution. Analyst. 2017, 142, 4067-4074. 9. Beleites, C.; Neugebauer, U.; Bocklitz, T.; Krafft, C.; Popp, J., Sample size planning for classification models. Anal. Chim. Acta. 2013, 760, 25-33.

10. Pavillon, N.; Hobro, A. J.; Akira, S.; Smith, N. I., Noninvasive detection of macrophage activation with single-cell resolution through machine learning. Proc. Natl. Acad. Sci. U S A. 2018, 115 (12), E2676E2685.

11. Anderson, M. J., A new method for non-parametric multivariate analysis of variance. Austral. Ecol. 2001, 26 (1), 32-46. 
12. Bocklitz, T.; Walter, A.; Hartmann, K.; Rosch, P.; Popp, J., How to pre-process Raman spectra for reliable and stable models? Anal. Chim. Acta. 2011, 704 (1-2), 47-56.

13. Lasch, P., Spectral pre-processing for biomedical vibrational spectroscopy and microspectroscopic imaging. Chemom. Intell. Lab. Syst. 2012, 117, 100-114.

14. Beier, B. D.; Berger, A. J., Method for automated background subtraction from Raman spectra containing known contaminants. Analyst. 2009, 134 (6), 1198-202.

15. Mazet, V.; Carteret, C.; Brie, D.; Idier, J.; Humbert, B., Background removal from spectra by designing and minimising a non-quadratic cost function. Chemom. Intell. Lab. Syst. 2005, 76 (2), 121-133. 